\title{
Ultrastable Natural Ester-Based Nanofluids for High Voltage Insulation Applications
}

Georgios D. Peppas, ${ }^{* \dagger}$ Aristides Bakandritsos, ${ }^{*}$ Vasilis P. Charalampakos, ${ }^{\S}$ Eleftheria C. Pyrgioti, ${ }^{\dagger}$ Jiri Tucek, ${ }^{\ddagger}$ Radek Zboril, ${ }^{\ddagger}$ Ioannis F. Gonos ${ }^{\#}$

${ }^{\dagger}$ Department of Electrical and Computer Engineering, University of Patras, 26504, Rio, Greece.

${ }^{+}$Regional Centre for Advanced Technologies and Materials, Department of Physical Chemistry, Faculty of Science, Palacky University in Olomouc, 17. listopadu 1192/12, 77146 Olomouc, Czech Republic.

${ }^{\S}$ Department of Electrical Engineering Technological Educational Inst. of Western Greece, Greece.

${ }^{\#}$ School of Electrical and Computer Engineering, National Technical University of Athens, Athens, Greece.

\section{Corresponding Author}

*E-mail: peppas@ece.upatras.gr.*E-mail: aristeidis.bakandritsos@upol.cz 


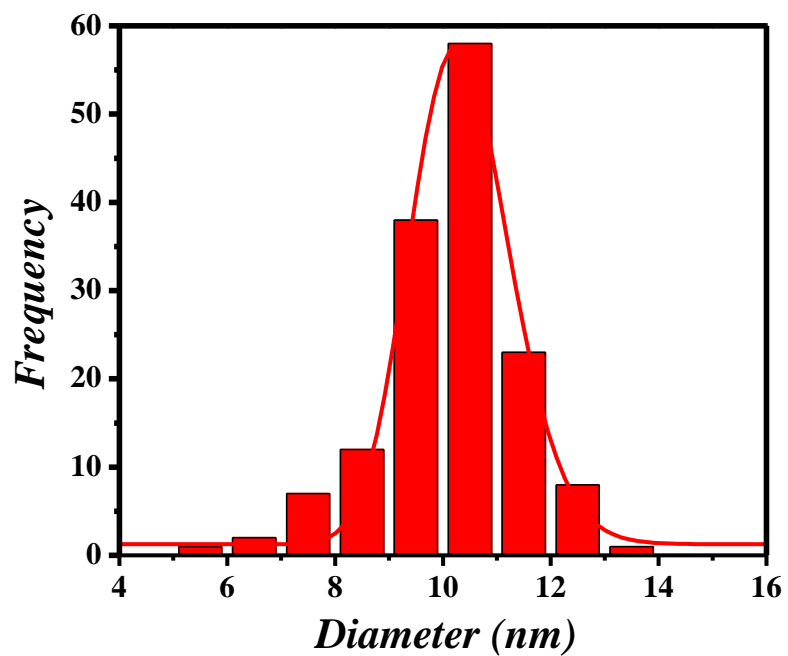

Figure S1. Size distribution diagram of the colloidal MIONs synthesized from the thermolytic route.

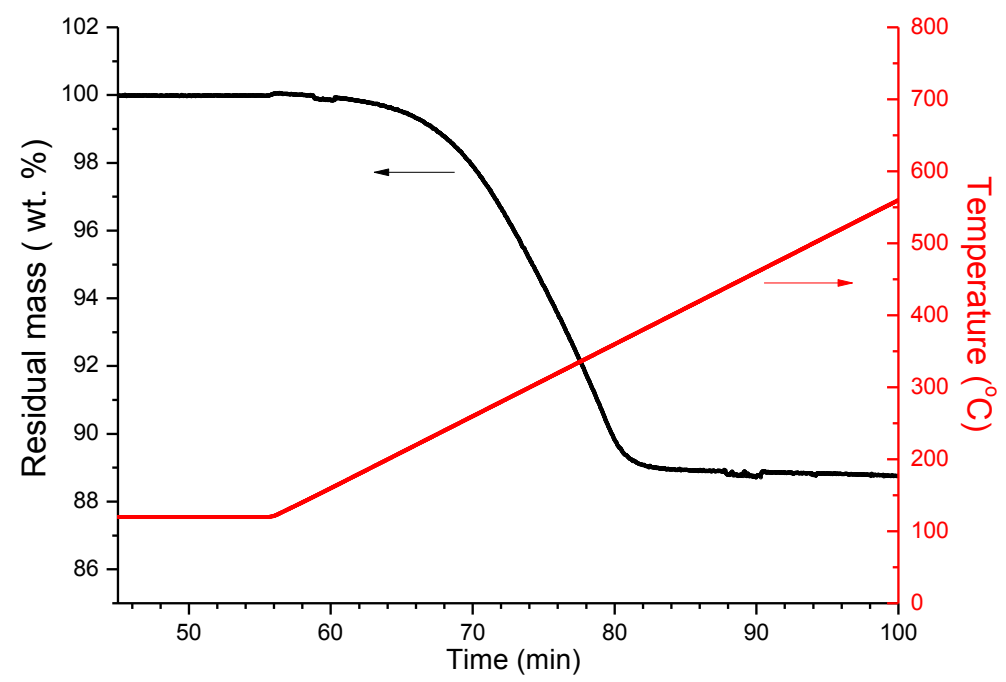

Figure S2. Thermogravimetric analysis of the synthesized colloidal MIONs after purification. The residual mass $(>88 \%)$ is ascribed to the non-combustible inorganic magnetic iron oxide nanoparticles, while the combustible component to the oleic-acid coating. 


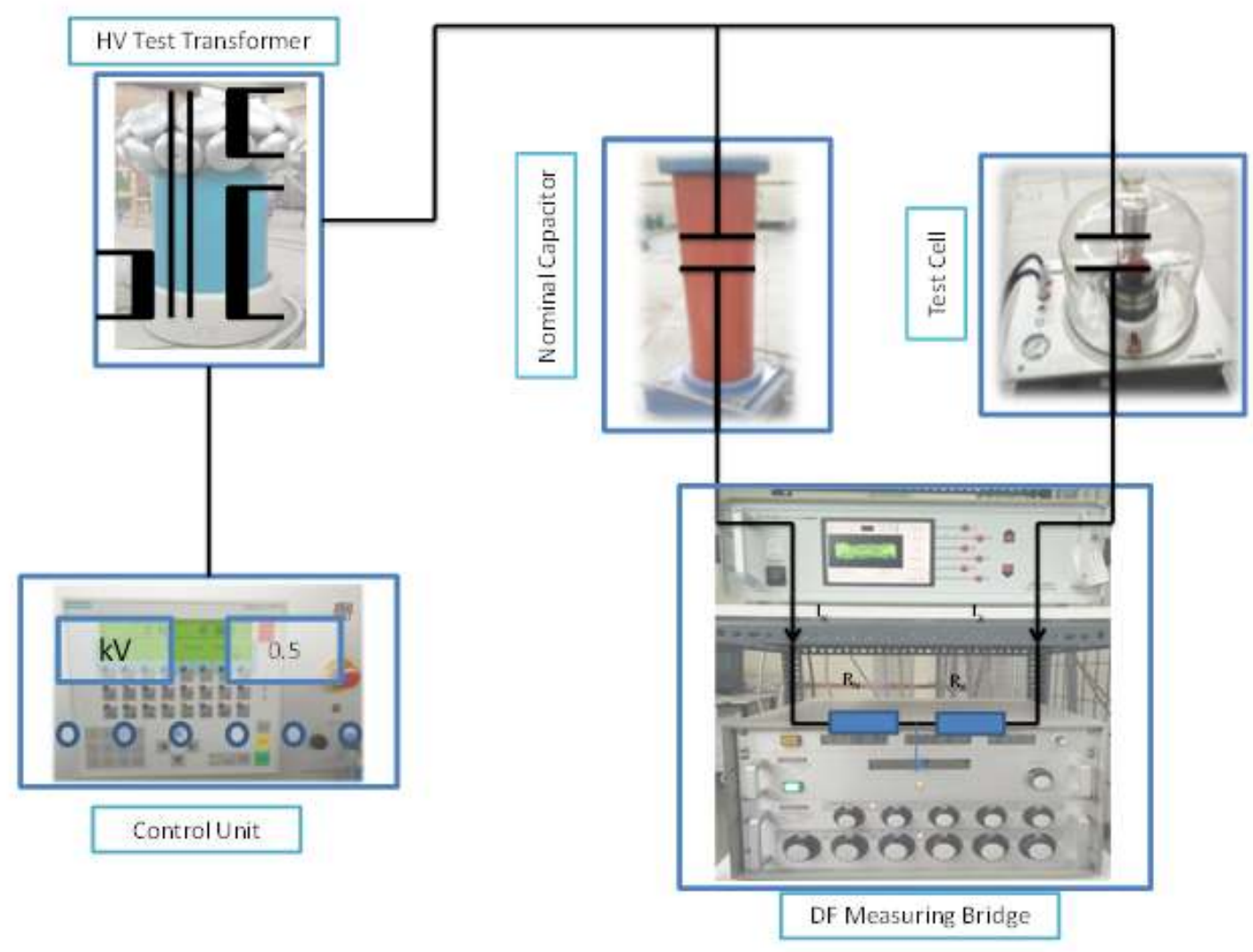

Figure S3. Schematic overview of the experimental setup (Glynne bridge) for the dissipation factor measurement, composed by a HighVolt GmbH control unit (Siemens Scada) a HighVolt GmbH HV test transformer PEOI 40/100 100kV, a Micafil standard capacitor SC100, a Tettex Instruments guard ring capacitor and a Haefely Bridge 470/471.

The FT-IR spectra of the oleic-acid coated MIONs synthesized in this work, and of the oleic-acid commercial MIONs are shown in Figure S4, termed as colNF and pNF, respectively. Bands from the oleic acid molecules are evident in both systems, although the oleic acid content in the commercial MIONs used in pNF is significantly lower. In Figure S4a the bands of the iron oxide lattice appear in both systems bellow $750 \mathrm{~cm}^{-1} .1,2,3$ At 2858 and $2928 \mathrm{~cm}^{-1}$ the symmetric and asymmetric stretching vibrations of the $-\mathrm{CH}_{2}$ groups from oleic acid are present. The weak band at $2960 \mathrm{~cm}^{-1}$ can be attributed to the vibration of the terminal $\mathrm{CH}_{3}$ group. ${ }^{3}$ In Figure $\mathrm{S} 4 \mathrm{~b}$ the $-\mathrm{CH}=\mathrm{CH}$ stretching mode from the double bond in oleic acid appears at $3010 \mathrm{~cm}^{-1}$ in both samples. The carbonyl band of oleic acid at $1711 \mathrm{~cm}^{-1}$ is mainly evident in the MIONs used in the colNF system, although it is also present in the commercial MIONs. This band is characteristic of free oleic acid, attributable to interdigitated bilayers of the molecules formed around the particles. ${ }^{3}$ Finally, the oleic acid molecules interacting with the MIONs give rise to oleate asymmetric and symmetric $-\mathrm{COO}^{-}$stretching frequencies. They appear at the region of 1400 to $1650 \mathrm{~cm}^{-1}$ for both MIONs systems. ${ }^{1,3}$ 

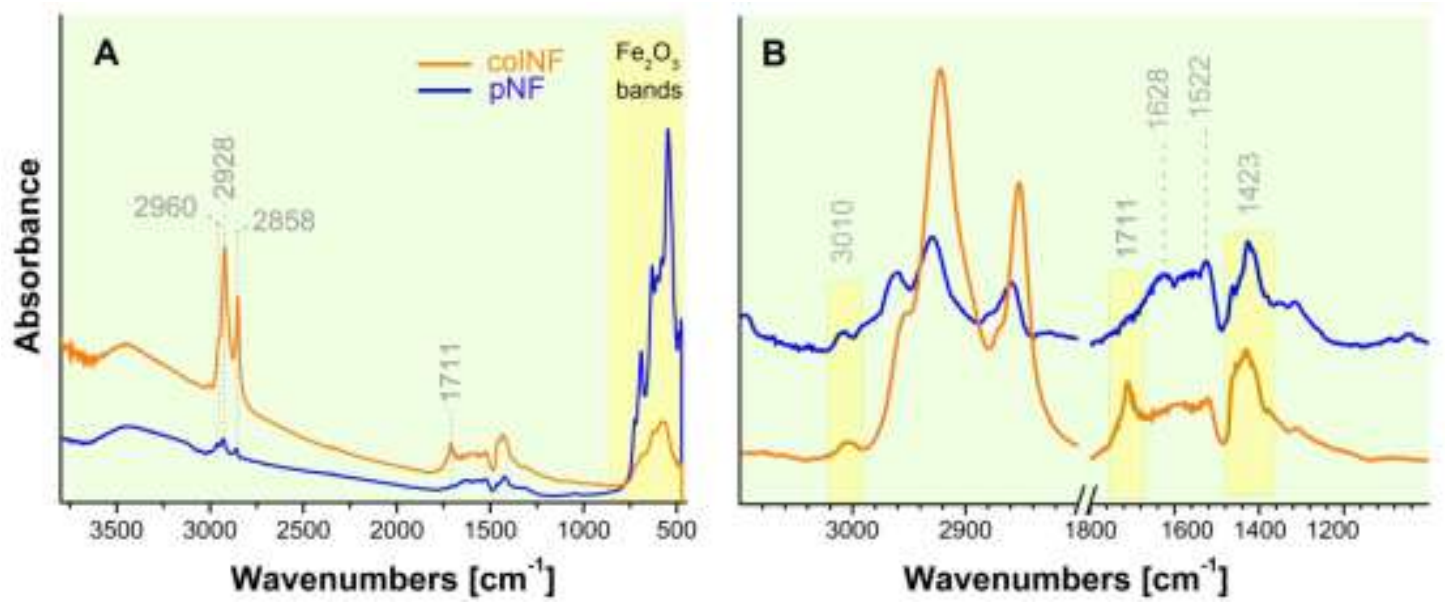

Figure S4. FT-IR spectra of the MIONs used in the colNF and in the pNF systems. (a) Full spectra of both MION systems. (b) Spectra of both MION systems, focused on the $2800-3100 \mathrm{~cm}^{-1}$ and 1000 $1800 \mathrm{~cm}^{-1}$ region.

The magnetic properties of the oleate-coated colloidal MIONs were monitored by measuring hysteresis loops at 5 and $300 \mathrm{~K}$ and recording ZFC/FC magnetization curves under an external magnetic field of $0.01 \mathrm{~T}$ (see Figure S5). At $5 \mathrm{~K}$, the isothermal magnetization curve shows a hysteresis, indicating that all the magnetic nanoparticles present in the colloid are in a magnetically ordered state with their superspins lying along the particular easy axis of magnetization energetically favored by the particle magnetic anisotropy. The values of the coercivity (i.e., 249 Oe, see inset in Figure S5) of the system falls within the range reported for bulk iron oxides of both $\gamma-\mathrm{Fe}_{2} \mathrm{O}_{3}$ and $\mathrm{Fe}_{3} \mathrm{O}_{4}$ nature. ${ }^{4}$ The saturation magnetization $\left(M_{\mathrm{S}}\right)$ of the oleate-coated colloidal MIONs reaches $\sim 47.5 \mathrm{Am}^{2} / \mathrm{kg}$, which is lower than that observed for bulk $\gamma-\mathrm{Fe}_{2} \mathrm{O}_{3}\left(\sim 60-80 \mathrm{Am}^{2} / \mathrm{kg}\right)$ and $\mathrm{Fe}_{3} \mathrm{O}_{4}\left(\sim 92-100 \mathrm{Am}^{2} / \mathrm{kg}\right){ }^{4}$ the reduced value of $M_{\mathrm{S}}$ can be well explained in terms of non-magnetic and/or diamagnetic response of oleate and other organic compounds in the colloid as the magnetization values are normalized to the total mass of the sample. Nevertheless, the saturation tendency of the system in low applied magnetic fields $(<1 \mathrm{~T})$ implies that the surface effects, typically emerging for nanoparticles with sizes of less than $15 \mathrm{~nm}$, do not significantly alter the magnetic properties of the nanoparticle assembly. ${ }^{5}$ At $300 \mathrm{~K}$, isothermal magnetization curve loses its hysteresis character (see bottom inset in Figure S5), a feature observed when magnetic nanoparticles enter the superparamagnetic regime and their superspins fluctuate between the orientations established by the easy axis of magnetization with the relaxation time much smaller than the measuring time of the PPMS device ( $\sim 10 \mathrm{~s})$. Upon warming, the passage from the magnetically blocked state to superparamagnetic regime is further evidenced from the maximum occurring at $~ 143$ $\mathrm{K}$ in the ZFC magnetization curve of the oleate-coated colloidal MIONs, ascribed to the average blocking temperature $\left(T_{\mathrm{B}}\right)$, i.e., the temperature at which the nanoparticles with the most probable size in the assembly enter superparamagnetic state. ${ }^{2}$ The ZFC and FC magnetization curves diverge from each other at $\sim 173 \mathrm{~K}$, which is known as the irreversibility 
temperature $\left(T_{\text {irr }}\right)$, signaling the onset of the magnetic blocking mechanism of superspins belonging to the largest nanoparticles in the system. ${ }^{5}$ In other words, the difference between $T_{\mathrm{B}}$ and $T_{\text {irr }}$ can be viewed as an indirect measure of the particle size distribution. As the values of $T_{\mathrm{B}}$ and $T_{\text {irr }}$ are close to each other, the oleate-coated colloidal MIONs show a rather narrow distribution in particle sizes as already witnessed from the representative TEM images. Thus, from the magnetization measurements, it can be concluded that the nanofluids made up of the oleate-coated MIONs are superparamagnetic at room temperature, reach magnetic saturation at low applied magnetic fields, and show strong magnetic response demonstrated by a high value of saturation magnetization. This property allows for exploitation of the magnetic properties of the nanoparticles without the need of using high magnetic fields.

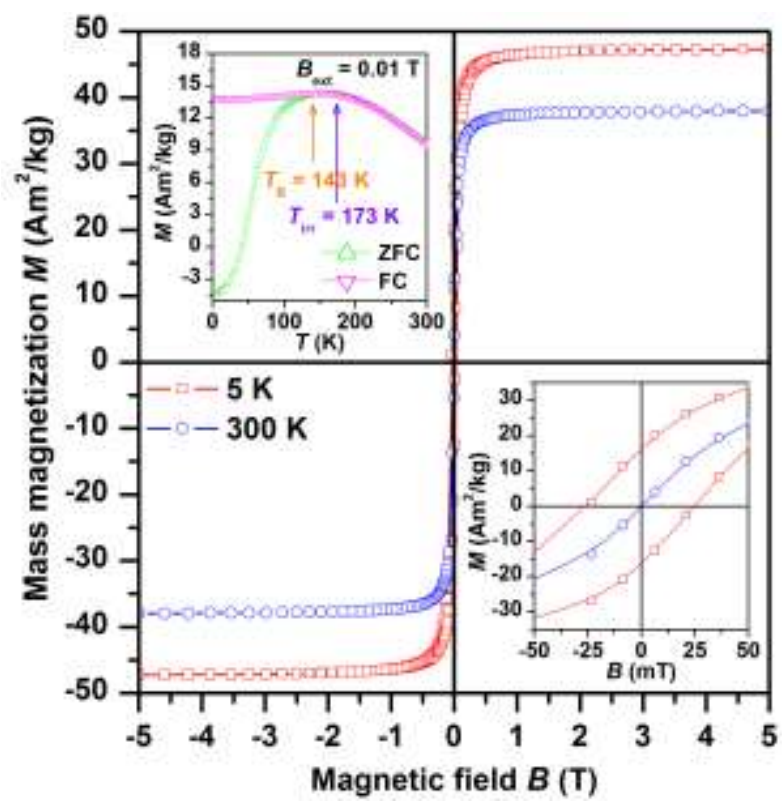

Figure S5. Hysteresis loops of the oleate-coated colloidal MIONs, recorded at a temperature of 5 and $300 \mathrm{~K}$. The bottom inset shows the profile of the hysteresis loops around the origin with a zoom on coercivity and remanent magnetization of the oleate-coated colloidal MIONs sample. The upper inset shows the ZFC/FC magnetization curves of the oleate-coated colloidal MIONs system, measured under an external magnetic field of $0.01 \mathrm{~T}$, with the blocking temperature $\left(T_{\mathrm{B}}\right)$ and irreversibility temperature $\left(T_{\text {irr }}\right)$ indicated.

\section{$\underline{\text { References }}$}

(1) Lee, Y.-J.; Jun, K.-W.; Park, J.-Y.; Potdar, H. S.; Chikate, R. C. A Simple Chemical Route for the Synthesis of $\gamma-\mathrm{Fe}_{2} \mathrm{O}_{3}$ Nano-Particles Dispersed in Organic Solvents via an Iron-hydroxy Oleate Precursor. J. Ind. Eng. Chem. 2008, 14, 38-44.

(2) Roca, A. G.; Marco, J. F.; Del, P. M.; Serna, C. J. Effect of Nature and Particle Size on Properties of Uniform Magnetite and Maghemite Nanoparticles. J. Phys. Chem. C 2007, 111, 18577-18584.

(3) Zboril, R.; Bakandritsos, A.; Mashlan, M.; Tzitzios, V.; Dallas, P.; Trapalis, C.; Petridis, D. OneStep Solid State Synthesis of Capped $\gamma-\mathrm{Fe}_{2} \mathrm{O}_{3}$ Nanocrystallites. Nanotechnology 2008, 19, 095602.

(4) Cornell, R. M. and Schwertmann, U. The Iron Oxides: Structure, Properties, Reactions, Occurences and Uses; $2^{\text {nd }}$ ed.; Wiley-VCH Verlag GmbH \& Co. KGaA: Weinheim, Germany, 2003; pp123,164.

(5) Dormann, J. L.; Fiorani, D.; Tronc, E. Magnetic relaxation in fine-particle systems. In Advances in Chemical Physic; Vol. 98, Prigogine, I., Rice, S. A., Eds.; John Wiley \& Sons: New York, U.S.A., 1997; pp 283-494. 